\title{
Managing Diabetic Macular Edema in Clinical Practice: Systematic Review and Meta-Analysis of Current Strategies and Treatment Options
}

This article was published in the following Dove Press journal:

Clinical Ophthalmology

\author{
Daniele Veritti (1) \\ Valentina Sarao id ${ }^{1,2}$ \\ Valentina Soppelsa (D) \\ Paolo Lanzetta (iD) 1,2 \\ 'Department of Medicine - \\ Ophthalmology, University of Udine, \\ Udine, Italy; ${ }^{2}$ Istituto Europeo di \\ Microchirurgia Oculare (IEMO), Udine, \\ Italy
}

Purpose: This meta-analysis aims to summarize 12-month best-corrected visual acuity (BCVA) outcomes in response to anti-vascular endothelial growth factor (VEGF) therapy and dexamethasone implant for the treatment of diabetic macular edema (DME) and to identify factors affecting treatment response using evidence generated from metaregression.

Methods: A systematic review of electronic databases was conducted to identify randomized controlled trials (RCTs) and real-life/observational studies that reported 12-month changes in BCVA in patients with DME on anti-VEGF or dexamethasone implant treatment in monotherapy. Study factors that were analyzed are baseline patient characteristics, study type, drug employed, number of injections and 12-month change in BCVA. Data were pooled in a random-effects meta-analysis with BCVA change as the main outcome. Meta-regression was conducted to assess the impact of multiple covariates.

Results: One-hundred-five heterogeneous study populations (45,032 eyes) were identified and included in the analysis. The use of anti-VEGFs and dexamethasone implant induced an overall increase of +8.13 ETDRS letters in BCVA at 12 months of follow-up. Metaregression provided evidence that mean BCVA change using anti-VEGFs was not statistically higher for RCTs $(p=0.35)$ compared to observational studies. Dexamethasone implant showed a trend for better results in observational studies over RCTs. Populations following a fixed aflibercept regimen performed better than those following a reactive treatment regimen. Mean BCVA gain was higher in younger populations $(\mathrm{p}<0.001)$, with lower baseline BCVA ( $<<0.0001)$ and longer diabetes duration $(p<0.0001)$, receiving a higher number of injections $(\mathrm{p}<0.0001)$.

Conclusion: Intravitreal therapy with anti-VEGFs or dexamethasone implant produces a significant improvement in BCVA at 12 months in patients with DME. Meta-regression identified the modifiable covariates that can be targeted in order to maximize functional results.

Keywords: aflibercept, anti-VEGF, bevacizumab, dexamethasone, diabetic macular edema, ranibizumab

\section{Introduction}

Diabetic macular edema (DME) is a sight-threatening disease and a major cause of blindness among people in working age. It affects about 7 million patients with diabetic retinopathy (DR). ${ }^{1}$ The prevalence of DME increases with duration of disease and stage of DR, approaching 30\% in adults who have had diabetes
Department of Medicine -

Ophthalmology, University of Udine, P.le Santa Maria della Misericordia I5, Udine, 33100 , Italy

Tel +390432559907

Fax +390432559904

Email paolo.lanzetta@uniud.it 
for more than 20 years and $71 \%$ of those with proliferative diabetic retinopathy. ${ }^{2,3}$

Grid and focal laser photocoagulation used to be the only evidence-based treatment modality to preserve vision in patients with DME. ${ }^{4}$ However, several decades of basic science research have revealed a growing and complex array of vascular permeability factors, such as vascular endothelial growth factor (VEGF), and proinflammatory mediators, providing the scaffold for identifying potential therapeutic targets. ${ }^{5}$ The introduction of intravitreal treatments has completely revolutionized the management of DME during the last decade. Anti-VEGF molecules, such as ranibizumab and aflibercept are commonly used as firstline therapy, while bevacizumab, a monoclonal antibody approved for the treatment of different types of cancer, is often employed in an off-label fashion in many countries. $^{6-11}$ An innovative, controlled-release, bioerodible dexamethasone implant was licensed for the treatment of DME in 2014. ${ }^{12}$ The current body of evidence on the efficacy and safety of anti-VEGF agents and dexamethasone implant is broad and steadily growing as randomized controlled trials (RCTs) and real-life/observational studies are completed. As clinical experiences have accumulated, it has become necessary to synthesize the updated results to provide information that can be useful in clinical practice. Therefore, we performed a systematic review and a meta-analysis of published RCTs and observational/reallife studies to sum up available evidence and to quantify the efficacy of intravitreal anti-VEGFs and dexamethasone implant for the treatment of DME. In addition, we aimed to identify patient and study factors that may affect the reporting of outcomes through a meta-regression model.

Specifically, the aim of this study is to respond to the following questions:

1. Is there a difference between results from RCTs and real-life/observational studies?

2. Is there a difference between results from RCTs and real-life/observational studies when analyzing drug classes?

3. Considering both RCTs and real-life/observational studies, does the treatment regimen influence the outcome when using anti-VEGFs?

4. Does the treatment regimen employed in real-life /observational studies influence the outcome when using anti-VEGFs?

5. If fixed regimen produces better outcomes, is this true for all anti-VEGFs?
6. Does the frequency of treatments influence the outcomes when using anti-VEGFs?

7. If frequency of injections influences the outcome, is this true for all anti-VEGFs?

8. Considering both RCTs and real-life/observational studies, which drug shows more favorable results in literature?

9. Which drug shows more favorable outcomes in literature, when considering only real-life/observational studies?

10. What are the baseline characteristics that significantly influence visual outcomes in real-life?

\section{Materials and Methods}

A multistage approach, including a systematic literature review (SLR), a meta-analysis, and a meta-regression, was used to determine the efficacy/effectiveness of intravitreal therapy in patients with DME.

\section{Systematic Literature Review}

A SLR of published studies including patients with DME receiving intravitreal ranibizumab, aflibercept, bevacizumab or dexamethasone implant with one-year follow-up was conducted. A systematic search of the PubMed, Medline, Embase, and Cochrane Library databases for relevant literature published was performed until May 15, 2020. The search strategy was centered on the amalgamation of medical subject headings and the keywords: "diabetic macular edema", "macular oedema", "macular edema", “anti-VEGF”, "DME”, "DMO", “aflibercept", "bevacizumab", "ranibizumab", "dexamethasone".

All search hits were imported into a spreadsheet, and data from eligible abstracts and full-text studies were extracted and selected on the basis of relevant populations, interventions, comparators, outcomes, and study design. The data extraction template was built using the MetaAnalyses and Systematic Reviews of Observational Studies guidelines, and the reporting of the SLR followed the Preferred Reporting Items for Systematic Reviews and Meta-Analyses (PRISMA) guidelines. ${ }^{13}$ Screening of title, abstract, and full text was performed using PRISMA guidelines. RCTs, and real-world prospective and retrospective clinical studies were included. Data from eligible articles meeting inclusion criteria were extracted independently by two authors (VSa and VSo) and disagreements were resolved by consensus. The risk of bias was evaluated both qualitatively and quantitatively using the Downs and Black checklist. The same two authors evaluated data 
and categorized articles obtained from the literature search to assess quality.

\section{Meta-Analysis}

A meta-analysis of the data identified from the SLR was undertaken. Inclusion criteria for the meta-analysis were studies enrolling DME patients who received monotherapy with ranibizumab, aflibercept, bevacizumab or dexamethasone implant, reported 52-week ( \pm 4 weeks) effectiveness outcomes. The primary objective of the meta-analysis was to derive a pooled estimate for effectiveness (defined as best-corrected visual acuity (BCVA) change from baseline to week 52 in Early Treatment Diabetic Retinopathy Study (ETDRS) letters). When necessary, variables of interest were converted: $\operatorname{logMAR}$ and decimal to ETDRS letters. Fixed-effects and random-effects models were used to produce estimates. Heterogeneity was assessed using the $\mathrm{I}^{2}$ statistic. Publication bias was evaluated by Egger's linear regression and visualized with funnel plots.

\section{Meta-Regression and Moderators Selection}

A meta-regression analysis was undertaken. Primary moderators were pre-selected based on existing evidence. Moderators included in the meta-regression were age at baseline, diabetes duration, baseline BCVA, baseline central retinal thickness (CRT), study type (RCT, real-life /observational study), treatment regimen, number of injections, and drug/drug class. The outcome variable assessed was mean BCVA change in ETDRS letters at 52 weeks ( \pm 4 weeks).

\section{Compliance with Ethics Guidelines}

This study is based on previously conducted studies and does not involve any new studies of human participants. This study did not require ethical approval as it did not involve human participants or animal subjects.

\section{Results}

\section{Study Selection}

The flowchart of the selection process is reported in Figure 1.

Our search resulted in 107 study titles that were screened for eligibility criteria.
Thirty-three papers were excluded based on failure to meet inclusion criteria, and 2 studies were ruled out on the basis of missing statistical parameters. The final analysis contained 105 heterogeneous patient populations described by 72 studies. ${ }^{14-80}$ The enrolled papers spanned 1 decade.

\section{Study Characteristics}

Characteristics of the 72 studies included for statistical analysis are shown in Table 1. All studies were composed of multiple heterogeneous study groups, which were treated as individual study populations in this analysis. A majority of studies were real-life/observational, which was defined as single-arm interventional designs and retrospective chart reviews. One-hundred-five heterogeneous study populations were identified. The size of the study populations varied widely, ranging from 6 to 15,273 eyes, with a total of 45,032 eyes included. Mean baseline BCVA ranged from 31 to 85 ETDRS letters. Mean baseline CRT ranged from 306 to $701 \mu \mathrm{m}$. Seventytwo studies were individually scored for their methodological quality using the Downs and Black checklist. Scores for methodological quality ranged from 14 to 20 , with an overall mean score of 17.1. Overall, reduced quality across studies can be attributed to inadequate reporting of blinding, loss to follow-up and characteristics of patients lost to follow-up, randomization, adjustment for confounding variables and estimates of random variability. Of the 72 studies included, 21 were randomized clinical trials, 22 were prospective cohort studies, and 29 were retrospective cohort studies.

\section{Meta-Analysis}

We found a high heterogeneity among included studies in this meta-analysis $\left(\mathrm{I}^{2}=96.542 \% ; \mathrm{p}<0.001\right)$ and a randomeffects model was chosen. A leave-one-out sensitivity analysis showed that none of the single studies had a significant effect on overall effect size. The metaanalysis showed an overall increase in BCVA at 12 months of +8.13 ETDRS letters (95\% CI: 7.26-9.00) (Forest plot is available as Figure S1).

\section{Meta-Regression Analyses}

Robust effect modification was found for several moderators. We employed a meta-regression approach to provide answers to clinically significant questions. 


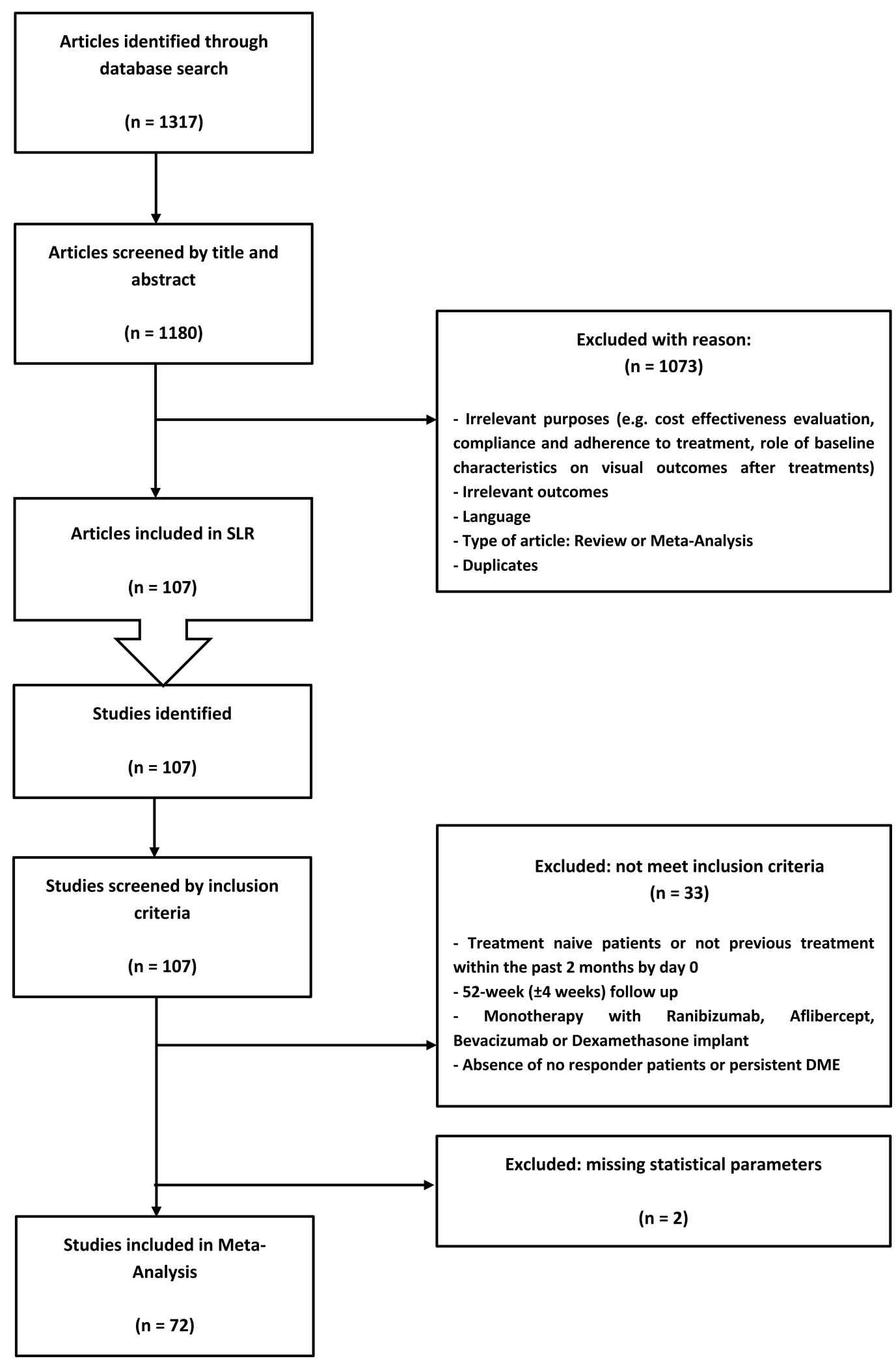

Figure I Flowchart of studies meeting inclusion and exclusion criteria from literature review. 
Table I Study Characteristics

\begin{tabular}{|l|l|l|l|l|}
\hline Study Type & RCT & Observational/Real-Life Studies & Prospective Studies & Retrospective Studies \\
\hline Eyes (populations) & $5404(37)$ & $39,628(68)$ & $5828(6 I)$ & $39,204(44)$ \\
\hline Drug & Ranibizumab & Aflibercept & Bevacizumab & Dexamethasone Implant \\
\hline Eyes (populations) & $12,322(43)$ & $10,767(30)$ & $19,067(14)$ & $2876(18)$ \\
\hline $\begin{array}{l}\text { Regimen } \\
\text { Eyes (populations) }\end{array}$ & $\begin{array}{l}\text { Fixed } \\
3831(32)\end{array}$ & $\begin{array}{l}\text { Pro-re-nata } \\
12,210(63)\end{array}$ & $\begin{array}{l}\text { Treat and Extend } \\
333(7)\end{array}$ & \\
\hline
\end{tabular}

Abbreviation: RCT, randomized controlled trial.

Is There a Difference Between Results from RCTs and Real-Life/Observational Studies?

Regression of difference in means on study type was not statistically significant $(\mathrm{p}=0.55)$, showing a coefficient of +0.55 ETDRS letters favouring RCTs over real-life/observational studies (CI 95\%: $-2.4 ;+1.3$ ).

\section{Is There a Difference Between Results from RCTs} and Real-Life/Observational Studies When Analyzing Drug Classes?

Regression of difference in means on study type for each anti-VEGF was not statistically significant $(p=0.35)$, showing a coefficient of +0.9 ETDRS letters favouring RCTs over real-life/observational studies (CI 95\%: $-2.7 ;+1.0)$. The same analysis performed for dexamethasone implant showed a trend for better results in real-life/observational studies over RCTs (coefficient +1.8 ETDRS letters; CI 95\%: -3.7 ; $+7.3 ; \mathrm{p}=0.5)$.

Considering Both RCTs and Real-Life/Observational Studies, Does the Treatment Regimen Influence the Outcome When Using Anti-VEGFs?

Regression of difference in means on regimen was statistically significant, favouring fixed regimen over pro-re-nata (PRN) regimen (coefficient +2.4 ETDRS letters; CI 95\%: +0.6; +4.1 ; $\mathrm{p}=0.009$ ), and treat and extend regimen (coefficient +3.6 ETDRS letters; CI 95\%: $+0.1 ;+7.2 ; \mathrm{p}=0.043$ ).

Does the Treatment Regimen Employed in Real-Life/ Observational Studies Influence the Outcome When Using Anti-VEGFs?

When considering only real-life/observational studies, regression of difference in means on regimen was not statistically significant. A slight trend towards better results with fixed regimen over PRN regimen (coefficient +0.4 ETDRS letters; CI 95\%: $-3.0 ;+3.9 ; \mathrm{p}=0.8)$, and treat and extend regimen (coefficient +2.3 ETDRS letters; CI $95 \%$ : $-3.2 ;+7.6 ; \mathrm{p}=0.4)$ was observed.

Fixed Regimen Produces Better Outcomes: Is This True for All Anti-VEGFs?

In ranibizumab-treated patients a trend towards better results for fixed regimen over PRN regimen (coefficient +0.7 ETDRS letters; CI 95\%: $-2.2 ;+3.7 ; \mathrm{p}=0.6$ ), and treat and extend regimen (coefficient +2.1 ETDRS letters; CI $95 \%$ : $-3.2 ;+7.6 ; p=0.4)$ was observed. Similarly, aflibercept-treated populations showed better results in studies employing fixed regimen over PRN regimen (coefficient +1.8 ETDRS letters; CI 95\%: $-1.3 ;+4.8 ; \mathrm{p}=0.2$ ), and treat and extend regimen (coefficient +4.5 ETDRS letters; $\mathrm{CI}$ 95\%: $-0.4 ;+9.4 ; \mathrm{p}=0.07)$. In bevacizumab-treated populations, regression of difference in means on regimen was not significantly different between fixed regimen and PRN regimen (coefficient +3.5 ETDRS letters; CI 95\%: -0.8 ; $+7.9 ; \mathrm{p}=0.1)$.

Does the Frequency of Treatments Influence the Outcomes When Using Anti-VEGFs?

Regression of difference in means on mean number of injections was highly statistically significant (coefficient +0.88 ETDRS letters; CI 95\%: $+0.57 ;+1.19 ; \mathrm{p}<0.0001$ ).

Frequency of Injections Influences the Outcome: Is This True for All Anti-VEGFs?

When considering all anti-VEGF agents, regression of difference in means on mean number of injections was highly statistically significant (coefficient +0.88 ETDRS letters; CI 95\%: $+0.56 ;+1.20 ; p<0.0001)$. For ranibizumab the coefficient is +1.08 ETDRS letters (CI 95\%: +0.57; $+1.59 ; \mathrm{p}<0.0001)$. For bevacizumab the coefficient is +0.99 ETDRS letters (CI 95\%: $+0.56 ;+1.42 ; \mathrm{p}<0.0001$ ). Regression of difference in means on mean number of injections was not statistically significant for aflibercept 
(coefficient +0.40 ETDRS letters; CI 95\%: $-0.22 ;+1.02$; $\mathrm{p}=0.21$ ).

Considering Both RCTs and Real-Life/Observational Studies, Which Drug Shows More Favorable Results in Literature?

Regression of difference in means on drug shows that the studies employing aflibercept reported significantly superior results over bevacizumab (coefficient +3.01 ETDRS letters; CI 95\%: $+0.10 ;+5.92 ; \mathrm{p}=0.04)$, and dexamethasone implant (coefficient +3.95 ETDRS letters; CI 95\%: $+1.25 ;+6.65 ; \mathrm{p}<0.01)$. The comparison with ranibizumab was not statistically significant (coefficient +2.01 ETDRS letters; CI 95\%: $-0.12 ;+4.15 ; \mathrm{p}=0.06)$.

\section{Which Drug Shows More Favorable Outcomes in Literature, When Considering Only Real-Life/ Observational Studies?}

Regression of difference in means on drug shows that the real-life/observational studies employing aflibercept reported not significantly different results over ranibizumab (coefficient +2.15 ETDRS letters; CI 95\%: -1.22 ; $+5.52 ; \mathrm{p}=0.21$ ), bevacizumab (coefficient +3.50 ETDRS letters; CI 95\%: -0.57 ; +7.57 ; $\mathrm{p}=0.09$ ), and dexamethasone implant (coefficient +3.56 ETDRS letters; CI 95\%: $-0.48 ;+7.61 ; \mathrm{p}=0.08)$.

What are the Baseline Characteristics That Significantly Influence Visual Outcomes in Real-Life? Regression of difference in means was significant on age (coefficient -0.54 ETDRS letters; CI 95\%: $-0.85 ;-0.23$; $\mathrm{p}<0.001$ ), diabetes duration (coefficient +0.98 ETDRS letters; CI 95\%: +0.54; +1.43; p<0.0001), baseline BCVA (coefficient -0.32 ETDRS letters; CI 95\%: -0.46 ; -0.18 ; $\mathrm{p}<0.0001)$. Regression of difference in means on baseline CRT was not statistically significant (coefficient +0.31 ETDRS letters; CI 95\%: $-0.33 ;+0.94 ; \mathrm{p}=0.34)$.

\section{Publication Bias and Sensitivity Analysis}

Visual inspection of funnel plots suggested an asymmetry in the meta-analysis. The presence of publication bias was also indicated by Egger's linear regression (intercept = 1.89, $\mathrm{p}=0.03$ ), but not by Begg's rank correlation test (Kendall's $\tau=0.07, p=0.26$ ). After adjustment of effect size for potential publication bias using the trim-and-fill correction, missing studies were imputed in the funnel plot, resulting in lower, albeit still highly significant estimate of pooled mean difference (adjusted $=+4.7$ ETDRS letters; CI 95\%: +3.7; +5.7; p<0.0001). Meta-analysis techniques "one-study-removed", used in the search for heterogeneity sources, and 'cumulative meta-analysis' (in inverted order of sample size), to assess the potential impact of a small-study effect, both showed negative results.

A sensitivity analysis in which studies were restricted to those published between 2010-2016 (41 populations, difference in means 8.391 CI95\% 7.115-9.667), and studies published between 2017-2020 (64 populations, difference in means 7.975 CI95\% 6.791-9.160) was performed. The results did not differ from the overall analysis.

\section{Discussion}

DME has long been recognized as a leading cause of vision loss in patients with diabetes. Extensive research has been underway for decades to understand the precise pathogenesis and potential treatment modalities to improve, stabilize and prevent DME. ${ }^{5}$ This study was performed to summarize the clinical evidence from RCTs and real-life/observational studies on visual outcomes of intravitreal pharmacologic approaches in the management of DME, obtaining a pooled estimate for visual acuity change from baseline to week 52 . The current meta-analysis, consisting of 45,032 eyes, is the largest and most comprehensive investigation to date that seeks to sum up the 12-month efficacy of intravitreal ranibizumab, aflibercept, bevacizumab and dexamethasone implant in treating DME. Overall, our results support the use of these drugs as effective treatment options for the management of DME, demonstrating that significant vision amelioration is achievable. The results showed an overall increase in visual acuity of approximately +8 ETDRS letters following 12 months of intravitreal therapy. However, a high variability exists between studies, as shown by the large variance in pooled effect size ( $p$ heterogeneity, <0.001). To elaborate on this matter, RCTs and real-life/observational studies were stratified into separate analyses. RCTs were calculated to have a pooled increase in visual acuity of +8.47 letters $(95 \%$ CI: 7.21-9.72) compared to baseline, and real-life/observational studies had pooled increase of +7.97 letters $(95 \%$ CI: 6.70-9.24). Overall, no statistically significant difference in terms of BCVA was detected between RCTs and real-life studies and, as expected, we found a high variability in real-life results. Even when stratified by drug classes (anti-VEGF agents and dexamethasone), differences in 1-year BCVA change between RCTs and real- 
life/observational studies remain not significant. This is in contrast to previous studies reporting that the real-world DME treatment outcomes with anti-VEGFs are meaningfully worse than those from RCTs. Specifically, patients with DME experienced a mean 1-year BCVA gain of approximately +5 letters in a clinical setting, while in the DRCR.net Protocol $\mathrm{T}$ trial, vision improved by a mean of $+13.3,+9.7$ and +11.2 at 1 year for eyes treated with aflibercept, bevacizumab and ranibizumab, respectively. ${ }^{9,19,80-82}$

The essential idea in a meta-analysis is that the estimates of the effect from previous studies of interest are pooled together, estimating a study-specific true effect. The observed heterogeneity in the estimates is attributed to between-study heterogeneity in true effects, and withinstudy sampling error. For the above-mentioned reasons, we believe that a random-effects approach is the best method to be used in our meta-analysis and metaregression. $^{83}$

When considering the results with dexamethasone implant, the visual outcomes surprisingly showed a trend to better performance in real-life studies $(+1.8$ letters $)$ than those observed in RCTs. Differences in DME duration and the possibility of retreating at an earlier stage in real life, as opposed to the fixed treatment regimens required for the larger RCT studies (every 6 months or 5 months) may explain this difference in results. ${ }^{46,84-88}$

Mounting evidence from the literature suggests that the frequency of anti-VEGF injections has an influence on the visual outcome when treating a DME patient. ${ }^{80,81,89,90}$ Our findings reinforce this assumption. Specifically, we found a statistically significant correlation between BCVA gain and the number of anti-VEGF injections. Each additional injection produces +0.88 letter gain at month 12 . However, these findings are not homogeneous for all anti-VEGF drugs.

Aflibercept seems to be the less sensitive agent to the frequency of injections (coefficient +0.40 ETDRS letters per injection). Conversely, ranibizumab and bevacizumab seem to be more dependent on the number of injections given per year (bevacizumab coefficient +0.99 , ranibizumab coefficient +1.08 ). This finding may be attributable to drug pharmacological properties and to the characteristics of the studies included in the analysis. Specifically, the variability in the number of injections is narrower in aflibercept studies than in those using ranibizumab and bevacizumab. This is mostly due to the fact that the majority of aflibercept studies use a proactive treatment scheduling (fixed or treat and extend) whereas a large proportion of the ranibizumab and bevacizumab studies apply a PRN approach which implies a larger variability in the number of injections. As known, the treatment regimen influences the final outcomes. ${ }^{89}$ In our metaregression, we found a trend to better outcomes when employing a fixed treatment regimen. Administrative and logistic factors may hinder therapeutic efficacy of PRN treatment regimen in a real-life scenario. Difficulty in scheduling appointments for treatment and monitoring visits is just an example of a real-world factor that may result in inferior actual clinical outcomes. A recently published comparison of DME trials shows that fixed dosing regimens or strict $\mathrm{PRN}$ regimens with clear retreatment criteria help to maximize the gains in BCVA, leading to optimal outcomes for patients. ${ }^{9}$ In contrast, the results from trials with less strict PRN treatment criteria show diminished BCVA gains and fewer injections in year 1 compared with trials with more rigorous regimens. ${ }^{6,9,24,25}$

When looking for baseline characteristics that may influence the outcome, we noted that the 12-month change in BCVA negatively correlated with baseline BCVA in patients with DME, which is consistent with prior studies noting a negative correlation between baseline BCVA and long-term BCVA change. ${ }^{91,92}$ This has been described as a "ceiling effect" in which the visual acuity gained by antiVEGF and dexamethasone implant use reaches a maximum over the time-course of treatment. Hence, patients with higher baseline visual acuity may experience a lesser degree of visual acuity gain due to starting with a visual acuity closer to the maximum benefit afforded by the treatment. ${ }^{93}$ Our meta-regression also revealed a decreasing trend in visual acuity change with increasing age. The negative correlation between age and BCVA change at 12 months observed is likely a manifestation of worsened outcomes at later age of presentation, when both the advanced progression of the disease and decreased response to treatment may result in inferior clinical outcomes. However, the correlation was not statistically significant which may be on account of the high variability between studies.

\section{Strengths and Limitations}

The present analysis has the following strengths: it provides an overview that is exhaustive and representative of the different therapeutic approaches used in clinical practice and in RCTs to manage patients with DME, we used a predefined search strategy and two independent 
reviewers conducted data extraction. Moreover, we conducted subgroup and sensitivity analysis. However, some important limitations should be noted. The main limitation of our meta-analysis is the quality of the included studies. Non-randomized studies exhibit a risk of bias. First, the heterogeneity among studies was high, possibly due to confounding variables as varying sample sizes, study designs, DME subtypes and treatment modalities. Actually, comparing RCTs with observational studies predisposes bias due to uncontrolled confounding. Moreover, this meta-analysis and meta-regression rely on papers which are not free from sample selection bias. In addition, the meta-analysis was based on aggregate data and not patient-level data, so it may be prone to ecological bias. Our findings are, consequently, an ecological association, based on aggregate measures in a highly selected population. They are not a causal association that can directly be extrapolated to an individual level. For these reasons, caution must be taken in assuming any particular form of quantitative relationship, which may change over time and with a greater number of studies. Summary estimates based on methodologically limited studies should not be over-interpreted and it must be noted that the best evidence synthesis to explore the impact of effect modifiers in a complex evidence network is a network meta-analysis. It allows both direct comparisons of interventions within randomized controlled trials and indirect comparisons across trials based on a common comparator.

\section{Conclusion}

In conclusion, the evidence for intravitreal therapy has been established in this meta-analysis to be highly favorable in the treatment of DME equally in clinical trials and real-world clinical settings. Frequent injections are required to preserve the outcomes of antiVEGF therapy. Increased injection frequency and younger age demonstrates a trend with improved outcomes.

\section{Disclosure}

Daniele Veritti is a consultant for Bayer, Novartis, and Roche. Valentina Sarao is a consultant for CenterVue and Roche. Paolo Lanzetta is a consultant for Allergan, Alcon, Bayer, Bausch \& Lomb, Novartis, CenterVue, Roche, and Topcon. The authors report no other conflicts of interest in this work.

\section{References}

1. Yau JWY, Rogers SL, Kawasaki R, et al. Global prevalence and major risk factors of diabetic retinopathy. Diabetes Care. 2012;35 (3):556-564. doi:10.2337/dc11-1909

2. Varma R, Bressler NM, Doan QV, et al. Prevalence of and risk factors for diabetic macular edema in the United States. JAMA Ophthalmol. 2014;132(11):1334-1350. doi:10.1001/jamaophthalmol.2014.2854

3. Klein R, Klein BE, Moss SE, et al. The Wisconsin epidemiologic study of diabetic retinopathy: IV. Diabetic macular edema. Ophthalmology. 1984;91(12):1464-1474. doi:10.1016/S01616420(84)34102-1

4. Early Treatment Diabetic Retinopathy Study Research Group. Early photocoagulation for diabetic retinopathy. ETDRS Report 9. Ophthalmology. 1991;98(Suppl 5):766-785. doi:10.1016/S01616420(13)38011-7

5. Kleinman ME, Baffi JZ, Ambati J. The multifactorial nature of retinal vascular disease. Ophthalmologica. 2010;224(Suppl 1):16-24. doi: $10.1159 / 000315152$

6. Mitchell P, Bandello F, Schmidt-Erfurth U, et al. The RESTORE study: ranibizumab monotherapy or combined with laser versus laser monotherapy for diabetic macular edema. Ophthalmology. 2011;118(4):615-625. doi:10.1016/j.ophtha.2011.01.031

7. Nguyen QD, Brown DM, Marcus DM, et al. Ranibizumab for diabetic macular edema: results from 2 Phase III randomized trials: RISE and RIDE. Ophthalmology. 2012;119(4):789-801. doi:10.1016/j.ophtha.2011.12.039

8. Brown DM, Nguyen QD, Marcus DM, et al. Long-term outcomes of ranibizumab therapy for diabetic macular edema: the 36-month results from two phase III trials: RISE and RIDE. Ophthalmology. 2013;120(10):2013-2022. doi:10.1016/j.ophtha.2013.02.034

9. Diabetic Retinopathy Clinical Research Network. Aflibercept, bevacizumab, or ranibizumab for diabetic macular edema. $N$ Engl J Med. 2015;372(13):1193-1203. doi:10.1056/NEJMoa1414264

10. Heier JS, Korobelnik JF, Brown DM, et al. Intravitreal aflibercept for diabetic macular edema: 148-week results from the VISTA and VIVID studies. Ophthalmology. 2016;123(11):2376-2385. doi:10.1016/j.ophtha.2016.07.032

11. Cai S, Bressler NM. Aflibercept, bevacizumab or ranibizumab for diabetic macular oedema: recent clinically relevant findings from DRCR.net Protocol T. Curr Opin Ophthalmol. 2017;28(6):636-643. doi:10.1097/ICU.0000000000000424

12. Boyer DS, Yoon YH, Belfort R, et al. Three-year, randomized, sham controlled trial of dexamethasone intravitreal implant in patients with diabetic macular edema. Ophthalmology. 2014;121(10):1904-1914. doi:10.1016/j.ophtha.2014.04.024

13. Moher D, Shamseer L, Clarke M, et al. Preferred reporting items for systematic review and meta-analysis protocols (PRISMA-P) 2015 statement. Syst Rev. 2015;4(1):1. doi:10.1186/2046-4053-4-1

14. Abouhussein MA, Gomaa AR. Aflibercept plus micropulse laser versus aflibercept monotherapy for diabetic macular edema: 1-year results of a randomized clinical trial. Int Ophthalmol. 2020;40 (5):1147-1154. doi:10.1007/s10792-019-01280-9

15. Baker CW, Glassman AR, Beaulieu WT, et al. Effect of initial management with aflibercept vs laser photocoagulation vs observation on vision loss among patients with diabetic macular edema involving the center of the macula and good visual acuity: a randomized clinical trial. JAMA. 2019;321(19):1880-1894. doi:10.1001/jama.2019.5790

16. Bhandari S, Nguyen V, Fraser-Bell S, et al. Ranibizumab or aflibercept for diabetic macular edema: comparison of 1-year outcomes from the fight retinal blindness! registry. Ophthalmology. 2019;127 (5):608-615. doi:10.1016/j.ophtha.2019.11.018

17. Polo RC, Sànchez CR, Guisado DMG, Luque JMD. Aflibercept for clinically significant diabetic macular edema: 12-month results in daily clinical practice. Clin Ophthalmol. 2018;12:99-104. doi:10.2147/OPTH.S154421 
18. Chen YX, Li XX, Yoon YH, et al. Intravitreal aflibercept versus laser photocoagulation in asian patients with diabetic macular edema: the VIVID-east study. Clin Ophthalmol. 2020;14:741-750. doi:10.2147/ OPTH.S235267

19. Ciulla TA, Bracha P, Pollack J, Williams DF. Real-world outcomes of anti-vascular endothelial growth factor therapy in diabetic macular edema in the United States. Ophthalmol Retina. 2018;2 (12):1179-1187. doi:10.1016/j.oret.2018.06.004

20. Curry BA, Sanfilippo PG, Chan S, Hewitt AW, Verma N. Clinical outcomes of a treat and extend regimen with intravitreal aflibercept injections in patients with diabetic macular edema: experience in clinical practice. Ophthalmol Ther. 2020;9(1):87-101. doi:10.1007/ s40123-019-00224-x

21. Do DV, Nguyen QD, Boyer D, et al. One-year outcomes of the da VINCI study of VEGF trap-eye in eyes with diabetic macular edema Ophthalmology. 2012;119(8):1658-1665. doi:10.1016/j.ophtha.2012. 02.010

22. Fouda SM, Bahgat AM. Intravitreal aflibercept versus intravitreal ranibizumab for the treatment of diabetic macular edema. Clin Ophthalmol. 2017;11:567-571. doi:10.2147/OPTH.S131381

23. Garweg JG, Stefanickova J, Hoyng C, et al. Vision-related quality of life in patients with diabetic macular edema treated with intravitreal aflibercept: the AQUA study. Ophthalmol Retina. 2019;3 (7):567-575. doi:10.1016/j.oret.2019.03.012

24. Glassman AR, Baker CW, Beaulieu WT, et al. Assessment of the DRCR retina network approach to management with initial observation for eyes with center-involved diabetic macular edema and good visual acuity: a secondary analysis of a randomized clinical trial. JAMA Ophthalmol. 2020;138(4):341-349. doi:10.1001/ jamaophthalmol.2019.6035

25. Hernández-Bel L, Cervera-Taulet E, Navarro-Palop C, CastroNavarro V, Chiarri-Toumit C, Montero-Hernández J. Sequential dexamethasone and aflibercept treatment in patients with diabetic macular edema: structural and functional outcomes at 52 weeks. Ophthalmologica. 2019;241(2):98-104. doi:10.1159/000489345

26. Kern C, Schiefelbein J, Fu JD, et al. Two year visual acuity and structural outcomes in patients with diabetic macular oedema treated with intravitreal aflibercept - a retrospective cohort study. Clin Ophthalmol. 2020;14:533-541. doi:10.2147/OPTH.S237586

27. Khattab AM, Hagras SM, AbdElhamid AH, Torky MA, Awad EA, Abdelhameed AG. Aflibercept with adjuvant micropulsed yellow laser versus aflibercept monotherapy in diabetic macular edema. Graefes Arch Clin Exp Ophthalmol. 2019;257(7):1373-1380. doi:10.1007/s00417-019-04355-6

28. Korobelnik JF, Daien V, Faure C, et al. Real-world outcomes following 12 months of intravitreal aflibercept monotherapy in patients with diabetic macular edema in France: results from the APOLLON study. Graefes Arch Clin Exp Ophthalmol. 2020;258(3):521-528. doi:10.1007/s00417-019-04592-9

29. Korobelnik JF, Do DV, Schmidt-Erfurth U, et al. Intravitreal aflibercept for diabetic macular edema. Ophthalmology. 2014;121 (11):2247-2254. doi:10.1016/j.ophtha.2014.05.006

30. Ozkaya A, Demir G, Kirmaci A. Comparison of aflibercept and ranibizumab in diabetic macular edema associated with subretinal detachment. Eur J Ophthalmol. 2019;30(2):363-369. doi:10.1177/ 1120672119827855

31. Ozsaygili C, Duru N. Comparison of intravitreal dexamethasone implant and aflibercept in patients with treatment-naive diabetic macular edema with serous retinal detachment. Retina. 2019;40 (6):1044-1052. doi:10.1097/IAE.0000000000002537

32. Pak KY, Shin JP, Kim HW, et al. One-year results of treatment of diabetic macular edema with aflibercept using the treat-and-extend dosing regimen: the VIBIM study. Ophthalmologica. 2020;243 (4):255-262. doi:10.1159/000504753
33. Schwarzer P, Ebneter A, Munk M, Wolf S, Zinkernagel MS. Oneyear results of using a treat-and-extend regimen without a loading phase with anti-VEGF agents in patients with treatment-naive diabetic macular edema. Ophthalmologica. 2019;241(4):220-225. doi:10.1159/000495623

34. Bilgic A, Sudhalkar A, Kodjikian L, et al. Pro re nata dexamethasone implant for treatment-naive phakic eyes with diabetic macular edema: a prospective study. Ophthalmol Retina. 2019;3(11):929-937. doi:10.1016/j.oret.2019.05.027

35. Borrelli E, Parravano M, Querques L, et al. One-year follow-up of ischemic index changes after intravitreal dexamethasone implant for diabetic macular edema: an ultra-widefield fluorescein angiography study. Acta Diabetol. 2019;57(5):543-548. doi:10.1007/s00592-01901435-1

36. Callanan DG, Loewenstein A, Patel SS, et al. A multicenter, 12-month randomized study comparing dexamethasone intravitreal implant with ranibizumab in patients with diabetic macular edema. Graefes Arch Clin Exp Ophthalmol. 2016;255(3):463-473. doi:10.1007/s00417-016-3472-1

37. Chhablani J, Bansal P, Veritti D, et al. Dexamethasone implant in diabetic macular edema in real-life situations. Eye (Lond). 2015;30 (3):426-430. doi:10.1038/eye.2015.246

38. Gillies MC, Lim LL, Campain A, et al. A randomized clinical trial of intravitreal bevacizumab versus intravitreal dexamethasone for diabetic macular edema: the BEVORDEX study. Ophthalmology. 2014;121(12):2473-2481. doi:10.1016/j.ophtha.2014.07.002

39. Iglicki M, Busch C, Zur D, et al. Dexamethasone implant for diabetic macular edema in naive compared with refractory eyes: the international retina group real-life 24-month multicenter study: the irgrel-dex study. Retina. 2018;39(1):44-51. doi:10.1097/ IAE.0000000000002196

40. Maggio E, Sartore M, Attanasio M, et al. Anti-vascular endothelial growth factor treatment for diabetic macular edema in a real-world clinical setting. Am J Ophthalmol. 2018;195:209-222. doi:10.1016/j. ajo.2018.08.004

41. Malcles A, Dot C, Voirin N, et al. Real-life study in diabetic macular edema treated with dexamethasone implant: the reldex study. Retina. 2017;37(4):753-760. doi:10.1097/IAE.00000000000 01234

42. Matonti F, Pommier S, Meyer F, et al. Long-term efficacy and safety of intravitreal dexamethasone implant for the treatment of diabetic macular edema. Eur J Ophthalmol. 2016;26(5):454-459. doi:10.5301/ejo.5000787

43. Menezo M, Roca M, Menezo V, Pascual I. Intravitreal dexamethasone implant ozurdex in the treatment of diabetic macular edema in patients not previously treated with any intravitreal drug: a prospective 12-month follow-up study. Curr Med Res Opin. 2019;35(12):2111-2116. doi:10.1080/03007995.2019.1652449

44. Ozkaya A, Algaroz C, Algaroz N, et al. Dexamethasone implant in pseudophakic and nonglaucomatous subgroup of diabetic macular edema patients: a real life experience. Eur J Ophthalmol. 2016;26 (4):351-355. doi:10.5301/ejo.5000725

45. Pareja-Ríos A, Ruiz-de la Fuente-rodríguez P, Bonaque-González S, López-Gálvez M, Lozano-López V, Romero-Aroca P. Intravitreal dexamethasone implants for diabetic macular edema. Int J Ophthalmol. 2018;11(1):77-82. doi:10.18240/ijo.2018.01.14

46. Rajesh B, Zarranz-Ventura J, Fung TA, et al. Safety of 6000 intravitreal dexamethasone implants. Br J Ophthalmol. 2019;104(1):39-46. doi:10.1136/bjophthalmol-2019-313991

47. Jabbarpoor Bonyadi MH, Baghi A, Ramezani A, Yaseri M, Soheilian M. One-year results of a trial comparing 2 doses of intravitreal ziv-aflibercept versus bevacizumab for treatment of diabetic macular edema. Ophthalmol Retina. 2017;2(5):428-440. doi:10.1016/j.oret.2017.09.010 
48. Koç I, Kadayifcilar S, Eldem B. Real-world results of intravitreal ranibizumab, bevacizumab, or triamcinolone for diabetic macular edema. Ophthalmologica. 2018;239(2-3):85-93. doi:10.1159/ 000481180

49. Kriechbaum K, Prager S, Mylonas G, et al. Intravitreal bevacizumab (Avastin) versus triamcinolone (Volon A) for treatment of diabetic macular edema: one-year results. Eye. 2014;28(1):10-16. doi:10.1038/eye.2013.242

50. Matsuda S, Tam T, Singh RP, et al. The impact of metabolic parameters on clinical response to VEGF inhibitors for diabetic macular edema. J Diabetes Complications. 2014;28(2):166-170. doi:10.1016/ j.jdiacomp.2013.11.009

51. Georgios M, Schranz M, Scholda C, et al. Response of retinal sensitivity to intravitreal anti-angiogenic bevacizumab and triamcinolone acetonide for patients with diabetic macular edema over one year. Curr Eye Res. 2020;45(9):1107-1113. doi:10.1080/ 02713683.2020.1712728

52. Nepomuceno AB, Takaki E, Paes de Almeida FP, et al. A prospective randomized trial of intravitreal bevacizumab versus ranibizumab for the management of diabetic macular edema. Am J Ophthalmol. 2013;156(3):502-513. doi:10.1016/j.ajo.2013.04.026

53. Michaelides M, Kaines A, Hamilton RD, et al. A prospective randomized trial of intravitreal bevacizumab or laser therapy in the management of diabetic macular edema (BOLT study) 12-month data: report 2. Ophthalmology. 2010;117(6):1078-1086. doi:10.1016/j. ophtha.2010.03.045

54. Sobaci G, Ozge G, Erdurman C, Durukan HA, Bayraktar ZM. Comparison of grid laser, intravitreal triamcinolone, and intravitreal bevacizumab in the treatment of diffuse diabetic macular edema. Ophthalmologica. 2011;227(2):95-99. doi:10.1159/000331322

55. Yaseri M, Zeraati H, Mohammad K, et al. Intravitreal bevacizumab injection alone or combined with triamcinolone versus macular photocoagulation in bilateral diabetic macular edema; application of bivariate generalized linear mixed model with asymmetric random effects in a subgroup of a clinical trial. J Ophthalmic Vis Res. 2014;9 (4):453-460. doi:10.4103/2008-322X.150818

56. Baget-Bernaldiz M, Romero-Aroca P, Bautista-Prez A, Mercado J. Multifocal electroretinography changes at the 1-year follow-up in a cohort of diabetic macular edema patients treated with ranibizumab. Doc Ophthalmol. 2017;135(2):85-96. doi:10.1007/ s10633-017-9601-2

57. Berger A, Sheidow T, Cruess AF, Arbour JD, Courseau AS, de Takacsy F. Efficacy/safety of ranibizumab monotherapy or with laser versus laser monotherapy in DME. Can $J$ Ophthalmol. 2015;50(3):209-216. doi:10.1016/j.jcjo.2014.12.014

58. Brown DM, Ou WC, Wong TP, Kim RY, Croft DE, Wykoff CC, for DAVE study group. Targeted retinal photocoagulation for diabetic macular edema with peripheral retinal nonperfusion: three-year randomized DAVE trial. Ophthalmology. 2018;125(5):683-690. doi:10.1016/j.ophtha.2017.11.026

59. Chatziralli I, Santarelli M, Patrao N, et al. Identification of time point to best define sub-optimal response' following intravitreal ranibizumab therapy for diabetic macular edema based on real-life data. Eye (Lond). 2017;31(11):1594-1599. doi:10.1038/eye.2017.111

60. Chen YP, Wu AL, Chuang CC, Chen SN. Factors influencing clinical outcomes in patients with diabetic macular edema treated with intravitreal ranibizumab: comparison between responder and non-responder cases. Sci Rep. 2019;9(1):10952. doi:10.1038/ s41598-019-47241-1

61. Epstein D, Amrèn U. Long-time outcome in patients treated with ranibizumab for diabetic macular edema: a 4-year study. Retina. 2018;38(1):183-186. doi:10.1097/IAE.0000000000001501

62. Ghanchi F, Hazel CA. South Asian diabetic macular oedema treated with ranibizumab (ADMOR)-real-life experience. Eye (Lond). 2016;30(1):133-138. doi:10.1038/eye.2015.209
63. Hrarat L, Fajnkuchen F, Boubaya M, et al. Outcomes after a 1-year treatment with ranibizumab for diabetic macular edema in a clinical setting. Ophthalmologica. 2016;236(4):207-214. doi:10.1159/ 000453006

64. Ishibashi $\mathrm{T}$, Li X, Koh A, et al. The REVEAL study: ranibizumab monotherapy or combined with laser versus laser monotherapy in Asian patients with diabetic macular edema. Ophthalmology. 2015;122(7):1402-1415. doi:10.1016/j.ophtha.2015.02.006

65. James DGP, Mitkute D, Porter G, Vayalambrone D. Visual outcomes following intravitreal ranibizumab for diabetic macular edema in a pro re nata protocol from baseline: a real-world experience. Asia Pac J Ophthalmology (Phila). 2019;8(3):200-205.

66. Karst SG, Lammer J, Mitsch C, Schmidt-Erfurth U. Detailed analysis of retinal morphology in patients with diabetic macular edema (DME) randomized to ranibizumab or triamcinolone treatment reply to the letter to the editor. Graefes Arch Clin Exp Ophthalmol. 2017;256(5):1039-1040. doi:10.1007/s00417-018-3902-3

67. Tso-Ting L, Chung-May Y, Chang Hao Y, Ho TC, Hsieh YT. Treatment outcomes and predicting factors for diabetic macular edema treated with ranibizumab - one-year real-life results in Taiwan. J Formos Med Assoc. 2019;118(1 Pt 1):194-202. doi:10.1016/j.jfma.2018.03.009

68. Li X, Dai H, Li X, et al. Efficacy and safety of ranibizumab $0.5 \mathrm{mg}$ in Chinese patients with visual impairment due to diabetic macular edema: results from the 12-month REFINE study. Graefes Arch Clin Exp Ophthalmol. 2019;257(3):529-541. doi:10.1007/s00417018-04213-x

69. Nguyen QD, Shah SM, Khwaia AA, et al. Two-year outcomes of the ranibizumab for edema of the macula in diabetes (READ-2) study. Ophthalmology. 2010;117(11):2146-2151. doi:10.1016/j.ophtha. 2010.08.016

70. Ozkaya A, Ozveren M, Demircan A. The real life data of ranibizumab use among the diabetic macular edema patients in Turkey: documenting the improvement with clinical optimization during three consecutive years. Saudi J Ophthalmol. 2018;32(3):175-179. doi:10.1016/j.sjopt.2018.04.005

71. Patrao NV, Antao S, Egan C, et al. Real-world outcomes of ranibizumab treatment for diabetic macular edema in a United Kingdom National Health Service setting. Am J Ophthalmol. 2016;172:51-57. doi:10.1016/j.ajo.2016.09.002

72. Payne JF, Wykoff CC, Lloyd Clark W, Bruce BB, Boyer DS, Brown DM, for TREX-DME study Group. Randomized trial of treat and extend ranibizumab with and without navigated laser for diabetic macular edema: TREX-DME 1 year outcomes. Ophthalmology. 2017;124(1):74-81. doi:10.1016/j. ophtha.2016.09.021

73. Prager SG, Lammer J, Mitsch C, et al. Analysis of retinal layer thickness in diabetic macular oedema treated with ranibizumab or triamcinolone. Acta Ophthalmol. 2018;96(2):e195-e200. doi:10.1111/aos.13520

74. Prünte C, Fajnkuchen F, Mahmood S, et al. Ranibizumab $0.5 \mathrm{mg}$ treat-and-extend regimen for diabetic macular oedema: the RETAIN study. $\mathrm{Br} J$ Ophthalmol. 2016;100(6):787-795. doi:10.1136/ bjophthalmol-2015-307249

75. Sato S, Shinoda H, Nagai N, et al. Predictive factors of better outcomes by monotherapy of an antivascular endothelial growth factor drug, ranibizumab, for diabetic macular edema in clinical practice. Medicine (Baltimore). 2017;96(16):e6459. doi:10.1097/MD.00000 00000006459

76. Takasago Y, Fujita T, Nakano Y, et al. Ranibizumab treatment improves diabetic macular oedema without influencing retinal oximetry parameters. Acta Ophthalmol. 2019;97(8):e1048-e1053. doi:10.1111/aos.14145

77. Talks SJ, Bhatia D, Menon G, et al. Randomised trial of wide-field guided PRP for diabetic macular oedema treated with ranibizumab. Eye (Lond). 2019;33(6):930-937. doi:10.1038/s41433-019-0342-1 
78. Tsai MJ, Hsieh YT, Peng YJ. Real-life experience of ranibizumab for diabetic macular edema in Taiwan. Int Ophthalmol. 2019;39 (7):1511-1522. doi:10.1007/s10792-018-0970-7

79. Xu Y, Rong A, Xu W, Niu Y, Wang Z. Comparison of 12-month therapeutic effect of conbercept and ranibizumab for diabetic macular edema: a real-life clinical practice study. BMC Ophthalmol. 2017;17 (1):158. doi:10.1186/s12886-017-0554-8

80. Ciulla TA, Pollack JS, Williams DF. Visual acuity outcomes and anti-VEGF therapy intensity in diabetic macular oedema: a real-world analysis of 28,658 patient eyes. $\mathrm{Br} J$ Ophthalmol. 2020; bjophthalmol-2020-315933. doi:10.1136/bjophthalmol-2020-315933

81. Blinder KJ, Dugel PU, Chen S, et al. Anti-VEGF treatment of diabetic macular edema in clinical practice: effectiveness and patterns of use (echo study report 1). Clin Ophthalmol. 2017;11:393-401. doi:10.2147/OPTH.S128509

82. Holekamp NM, Campbell J, Almony A, et al. Vision outcomes following anti-vascular endothelial growth factor treatment of diabetic macular edema in clinical practice. Am J Ophthalmol. 2018;191:83-91. doi:10.1016/j.ajo.2018.04.010

83. Tufanaru C, Munn Z, Stephenson M, Aromataris E. Fixed or random effects meta-analysis? Common methodological issues in systematic reviews of effectiveness. Int $J$ Evid Based Healthc. 2015;13 (3):196-207. doi:10.1097/XEB.0000000000000065

84. Kodjikian L, Bellocq D, Mathis T. Pharmacological management of diabetic macular edema in real-life observational studies. Biomed Res Int. 2018;2018:8289253. doi:10.1155/2018/8289253

85. Rosenblatt A, Udaondo P, Cunha-Vaz J, et al. A collaborative retrospective study on the efficacy and safety of intravitreal dexamethasone implant (Ozurdex) in patients with diabetic macular edema. Ophthalmology. 2020;127(3):377-393. doi:10.1016/j. ophtha.2019.10.005

86. Veritti D, Sarao V, Galiazzo F, Lanzetta P. Early effects of dexamethasone implant on macular morphology and visual function in patients with diabetic macular edema. Ophthalmologica. 2017;238 (1-2):100-105. doi:10.1159/000475889
87. Sarao V, Veritti D, Furino C, et al. Dexamethasone implant with fixed or individualized regimen in the treatment of diabetic macular oedema: six-month outcomes of the UDBASA study. Acta Ophthalmol. 2017;95(4):e255-e260. doi:10.1111/aos.13395

88. Al-Khersan H, Hariprasad SM, Chhablani J; for Dex Implant Study Group. Early response to intravitreal dexamethasone implant therapy in diabetic macular edema may predict visual outcome. Am J Ophthalmol. 2017;184:121-128. doi:10.1016/j.ajo.2017.10.004

89. Wecker T, Ehlken C, Bühler A, et al. Five-year visual acuity outcomes and injection patterns in patients with pro-re-nata treatments for AMD, DME, RVO and myopic CNV. Br J Ophthalmol. 2017;101 (3):353-359. doi:10.1136/bjophthalmol-2016-308668

90. Lanzetta P, Loewenstein A, for Vision Academy Steering C. Fundamental principles of an anti-VEGF treatment regimen: optimal application of intravitreal anti-vascular endothelial growth factor therapy of macular diseases. Graefes Arch Clin Exp Ophthalmol. 2017;255(7):1259-1273. doi:10.1007/s00417-017-3647-4

91. Brown DM, Schmidt-Erfurth U, Do DV, et al. Intravitreal aflibercept for diabetic macular edema: 100-week results from the VISTA and VIVID studies. Ophthalmology. 2015;122(10):2044-2052. doi:10.1016/j.ophtha.2015.06.017

92. Heier JS, Bressler NM, Avery RL, et al. Comparison of aflibercept, bevacizumab, and ranibizumab for treatment of diabetic macular edema: extrapolation of data to clinical practice. JAMA Ophthalmol. 2016;134(1):95-99. doi:10.1001/jamaophthalmol.2015.4110

93. Dugel PU, Hillenkamp J, Sivaprasad S, et al. Baseline visual acuity strongly predicts visual acuity gain in patients with diabetic macular edema following anti-vascular endothelial growth factor treatment across trials. Clin Opthalmol. 2016;10:1103-1110. doi:10.2147/ OPTH.S100764
Clinical Ophthalmology

\section{Publish your work in this journal}

Clinical Ophthalmology is an international, peer-reviewed journal covering all subspecialties within ophthalmology. Key topics include: Optometry; Visual science; Pharmacology and drug therapy in eye diseases; Basic Sciences; Primary and Secondary eye care; Patient Safety and Quality of Care Improvements. This journal is indexed on PubMed
Dovepress

Central and CAS, and is the official journal of The Society of Clinical Ophthalmology (SCO). The manuscript management system is completely online and includes a very quick and fair peer-review system, which is all easy to use. Visit http://www.dovepress.com/ testimonials.php to read real quotes from published authors. 